\title{
Protective effect of fucoidan against tacrolimus-induced nephrotoxicity in LLC-PK1 cells
}

Hyuk Jai Jang

Division of Transplantation, Department of Surgery, Gangneung Asan Hospital, Gangneung, Korea

Background: Tacrolimus (FK506) is an immunosuppressant agent that is frequently used to prevent rejection of solid organs upon transplant. However, nephrotoxicity due to apoptosis and inflammatory response mediated by FK506 limit its usefulness. Fucoidan (FUC) is an antioxidant and anti-inflammatory sulfated polysaccharide compound from brown seawood. In the present study, the protective effect of FUC against FK506-induced damage in LLC-PK1 pig kidney epithelial cells was investigated.

Methods: LLC-PK1 cells were exposed to FK506 with FUC and cell viability was measured. Western blotting and RT-PCR analyses evaluated protein or gene expression of $\mathrm{HO}-1$, superoxide dismutase-2 (SOD-2), Bcl-2, Bax, caspase-3, inducible nitric oxide synthase (iNOS), and cyclooxygenase-2 (COX-2) expression were assessed. The number of apoptotic cells was measured using an annexin V/PI staining with flow cytometry.

Results: Reduction in cell viability by 50 mM FK506 was ameliorated significantly by cotreatment with FUC. COX-2, iNOS, Bax and cleaved caspase-3, increased markedly in LLC-PK1 cells treated with FK506 and significantly decreased after cotreatment with FUC. HO-1, SOD-2, and Bcl-2 significantly increased in LLC-PK1 cells treated with FK506 after cotreatment with FUC. Moreover, flow cytometry assay showed that apoptotic cell death was increased by FK506 treatment, whereas it was decreased after cotreatment with FUC.

Conclusions: These results collectively provide therapeutic evidence that FUC ameliorates the FK506-induced renal damage via antioxidant effect and inhibiting apoptosis and inflammation.

Corresponding author: Hyuk Jai Jang

E-mail: d990081@gmail.com 